\title{
Noninvasive gene transfer to the lung for systemic delivery of therapeutic proteins
}

\author{
Alberto Auricchio, ${ }^{1}$ Erin O'Connor, ${ }^{1}$ Daniel Weiner, ${ }^{2}$ Guang-Ping Gao, ${ }^{1}$ \\ Markus Hildinger, ${ }^{1}$ Lili Wang, ${ }^{1}$ Roberto Calcedo, ${ }^{1}$ and James M. Wilson ${ }^{1}$
} ${ }^{1}$ Institute for Human Gene Therapy, The Wistar Institute, Department of Medicine, and
${ }^{2}$ Division of Pulmonary Medicine, Department of Pediatrics, The Children's Hospital of Philadelphia and
the University of Pennsylvania, Philadelphia, USA

\begin{abstract}
This study evaluates the use of vectors based on adeno-associated viruses (AAVs) to noninvasively deliver genes to airway epithelial cells as a means for achieving systemic administration of therapeutic proteins. We intranasally delivered AAV vectors to mice in which the same AAV2 genome encoding a cellular marker was packaged in capsids from AAV1, 2, or 5 (AAV2/1, AAV2/2, or AAV2/5, respectively). Gene expression levels achieved in both airways and alveoli were higher with AAV2/5 than with AAV2/1 and were undetectable with AAV2/2. The same set of vectors encoding a secreted therapeutic protein, erythropoietin (Epo), under the control of a lung-specific promoter (CC10) was intranasally delivered to mice, resulting in polycythemia with the highest levels of serum Epo obtained with AAV2/5 vectors. After a single intranasal administration of this vector, secretion of Epo was documented for 150 days. Similarly, intranasal administration of an AAV2/5-CC10-factor IX vector resulted in secretion of functional recombinant protein in the bloodstream of hemophiliac, factor IX-deficient mice. In addition, we demonstrate successful readministration of AAV2/5 to the lung 5 months after the first delivery of the same vector. In conclusion, we show that intranasal administration of AAV vectors results in efficient gene transfer to the lung only when the vector contains the AAV5 capsid and that this noninvasive route of administration results in sustained secretion of therapeutic proteins in the bloodstream.

J. Clin. Invest. 110:499-504 (2002). doi:10.1172/JCI200215780.
\end{abstract}

\section{Introduction}

Lung diseases such as cystic fibrosis and $\alpha$-1-antitrypsin are attractive candidates for gene replacement therapy, for several reasons. Airway epithelial cells are easily accessed by noninvasive aerosol delivery of vectors. Additionally, the lung may be used as a depot site for systemic and local delivery of secreted therapeutic proteins. This is facilitated by a large surface area (50 $\mathrm{m}^{2}$ ) and an extensive pulmonary-capillary network. This strategy has been exploited for delivery of antibiotics and is being explored for the direct delivery of metabolic hormones with systemic effects, such as insulin and growth hormone (1). A number of vector systems have targeted epithelial cells of the lung following aerosol delivery (2-4).

Received for publication April 24, 2002, and accepted in revised form June 5, 2002.

Address correspondence to: James M. Wilson, 204 Wistar Institute, 3601 Spruce Street, Philadelphia, Pennsylvania 19104-4268, USA. Phone: (215) 898-3000; Fax: (215) 898-6588;

E-mail: wilsonjm@mail.med.upenn.edu.

Conflict of interest: J.M. Wilson holds equity in Targeted Genetics Corp.

Nonstandard abbreviations used: adeno-associated virus (AAV); erythropoietin (Epo); rhesus monkey erythropoietin (rhEpo); factor IX (FIX); canine factor IX (cFIX); genome copy (GC); activated partial thromboplastin time (aPTT); human placental alkaline phosphatase (hPALP); enhanced green fluorescent protein (EGFP).
Vectors based on adenovirus have generally demonstrated limited efficiency and persistence of transgene expression in the lung, likely due to a combination of a lack of receptors on the apical surface of the airway epithelial cells, and a robust inflammatory response. Vectors based on adeno-associated viruses (AAVs) are promising delivery vehicles for achieving stable gene expression (5), because of the inherent stability of the vector genome and its ability to evade the immune system. Six primary isolates of AAV have been described to date (6-11). Vectors can be constructed by packaging the same AAV2 genome into capsids from different serotypes, which allows for easy alteration of tropism. Vectors based on different serotypes will allow successful gene transfer in the setting of pre-existing neutralizing antibodies that result from a natural infection or previous exposure to vectors (12). Although there has been some success using AAV2-based vectors in the lung (13), most experiments with AAV2 gene delivery to airway epithelial cells from the luminal side have been disappointing $(14,15)$. Vectors based on AAV5 or 6 (the capsid from serotype 6 is nearly identical to that from serotype 1 , differing in only six amino acids) have been shown to enter from the apical side and to more efficiently transduce airway cells $(13,16)$. Capsid proteins from AAV5 have been shown to bind to sialic acid (17), a component of a putative specific receptor present on the apical surface of airway epithelial cells. 
This study sought to evaluate the potential of AAVmediated gene transfer to the lung as a means of achieving stable levels of systemically secreted proteins that are easily measured, physiologically active, and potentially therapeutic.

\section{Methods}

Vector construction. Rat lung genomic DNA (Clontech Laboratories Inc., Palo Alto, California, USA) was used as a template for PCR amplification of the Clara Cell 10 (CC10) promoter. The resulting PCR product was cloned into the pCR 2.1-Topo vector (Invitrogen, Carlsbad, California, USA) using the Topo TA Cloning kit according to the manufacturer's instructions. This plasmid, containing the first $500 \mathrm{bp}$ of the rat CC10 promoter, was then used as a second template for PCR amplification of the desired 320-bp CC10 promoter fragment (18). The PCR product was cloned into pCR 2.1-Topo. pAAV2.1 CMV lacZ 2 (19) was digested to remove the CMV promoter, and the CC10 promoter, isolated from Topo, was inserted to produce pAAV2.1 CC10 lacZ 2.

pAAV2.1 CC10 rhEpo 3 was cloned by inserting the rhEpo 3, obtained by digestion of pAAV2.1 CMV rhEpo 3 (19), into pAAV2 CC10 lacZ 2. Rhesus monkey erythropoietin (rhEpo) was used because of the availability of an ELISA specific for human Epo that strongly cross-reacts with rhEpo.

pAAV2.1 CC10 cFIX, containing the coding sequence for canine factor IX (cFIX), was cloned as follows: We first prepared pAAV2.1 CC10 mInsII 3 by removing the TBG promoter of pAAV2.1 TBG mInsII 3 (19) and replacing it with the CC10 promoter discussed above. The cFIX WPRE fragment from pAAV2.1 LSP cFIX WPRE pA (20) was introduced into the pAAV2.1 CC10 mInsII 3 backbone to produce pAAV2.1 CC10 FIX WPRE. The cFIX homologue was used because of its previously demonstrated ability to correct FIX deficiency in the mouse model of the disease.

The sequence of the pAAV2-CB-human placental alkaline phosphatase (pAAV2-CB-hPALP) containing the chicken $\beta$-actin enhancer and CMV minimal promoter (21), as well as of any other plasmids used in this study, is electronically available upon request to the corresponding author.

Vector production and characterization. Recombinant AAV2, AAV2/5, and AAV2/1 viruses were produced according to protocols described elsewhere $(6,19,22)$. For each viral preparation, physical titers (genome copies [GCs] per ml) were determined by PCR quantification using TaqMan (Perkin Elmer, Foster City, California, USA) (23). The infectivity of each viral preparation was measured by the infectious center assay (ICA) (24) using 293 cells. ICA ratios (GCs per infectious unit) for the rhEpo/cFIX and alkaline phosphatase viruses, respectively, were 200 and 48 for the AAV2 vectors, 800 and 83 for the AAV2/5 vectors, and 3,000 and 286 for the AAV2/1 vectors.

Vector administration to animals. Female NCR nude mice and male C57BL/6 mice were purchased from
Taconic (Germantown, New York, USA). FIX $X^{-/-}$mice on a C57BL/ 6 background (25) were bred at the Wistar Institute Animal Facility. Mice 6-8 weeks old were anesthetized in groups of four or five with intraperitoneal ketamine and xylazine, suspended vertically, and exposed to $50 \mu \mathrm{l}$ of virus per mouse by nasal instillation. The specific vector doses used in the experiments are described in the text.

Analysis of transgene expression in lung. At day 60 following vector administration, animals receiving AAVCC10-lacZ vector were sacrificed, and their lungs were inflated with OCT compound (Sakuraus Finetek USA Inc., Torrance, California, USA), frozen, cryosectioned, and stained for lac $Z$ expression (26). In the readministration experiment, lungs were harvested 28 days after vector delivery and homogenized. Protein concentration in each sample was standardized to $2 \mathrm{mg} / \mathrm{ml}$ before measurement of the $\beta$-galactosidase concentration using a commercially available ELISA (Roche Molecular Biochemicals, Mannheim, Germany) according to manufacturer's instructions.

In the experiments involving alkaline phosphatase expression, animals were euthanized 28 days after vector administration, at which point the left lung was immediately homogenized in lysis buffer (Reporter Gene Assay Lysis Buffer; Roche Molecular Biochemicals, Indianapolis, Indiana, USA) containing protease inhibitor (Complete Protease Inhibitor Cocktail; Roche Molecular Biochemicals, Indianapolis, Indiana, USA). The homogenate was centrifuged to remove tissue debris, and the supernatant was stored for analysis. The right lung and trachea were embedded in OCT compound, frozen, and cryosectioned. The slides were fixed with $0.5 \%$ glutaraldehyde and incubated in $1 \mathrm{mM} \mathrm{MgCl}_{2}$ at $65^{\circ} \mathrm{C}$ to inactivate endogenous alkaline phosphatase. After rinsing, the slides were stained with alkaline phosphatase buffer (1.2 g Tris-base, $0.584 \mathrm{~g} \mathrm{NaCl}, 1.017 \mathrm{~g}$ $\mathrm{MgCl}_{2}$ in $100 \mathrm{ml}$ water, $\left.\mathrm{pH} 9.5\right), 220 \mu \mathrm{l}$ nitro-blue tetrazolium chloride, and $165 \mu 1$ 5-bromo-4-chloro3 -indolylphosphate toluidine. Sections were incubated for 30 minutes, rinsed, and counterstained with nuclear fast red. The lung homogenate was quantitatively analyzed for placental alkaline phosphatase using two separate methods according to the manufacturers' protocols (SEAP Reporter Gene Assay, Roche Molecular Biochemicals, Indianapolis, Indiana, USA; and Innotest hPALP ELISA, Innogenetics, Ghent, Belgium).

Analysis of proteins secreted in serum and lung. Serum samples were analyzed at designated time points for human Epo using the Quantikine Human EPO Immunoassay (R\&D Systems Inc., Minneapolis, Minnesota, USA). Hematocrit levels were also measured in these animals by microcapillary centrifugation. In the experiments involving FIX secretion, plasma samples were collected and analyzed using ELISA as described (27) and activated partial thromboplastin times (aPTTs). 

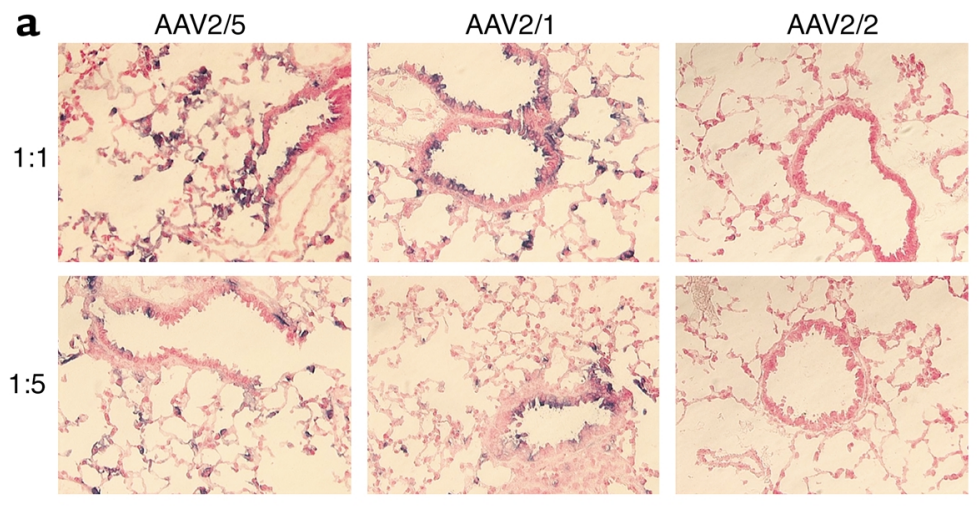

b

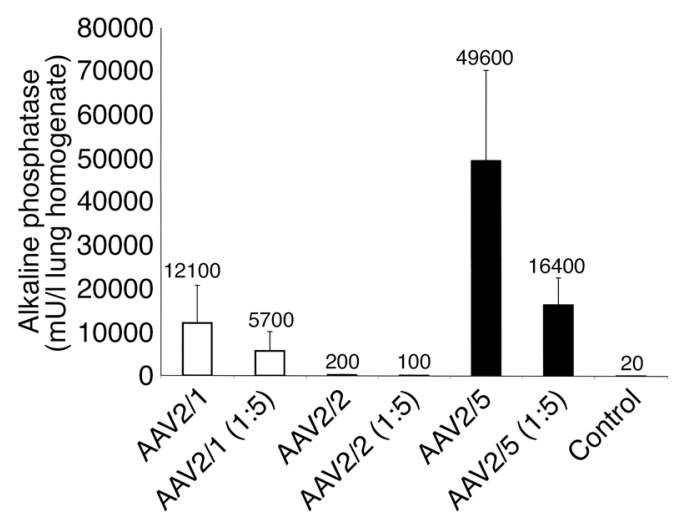

Figure 1

Transduction of murine lung with vectors based on various AAV serotypes encoding hPALP. Vectors were intranasally administered undiluted

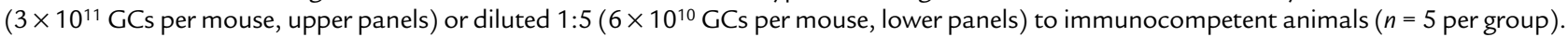
(a) Histochemical detection of PALP in the lung 4 weeks after vector delivery. Positive cells are present in both alveolar and airway cells when $\mathrm{AAV} 2 / 5$ and AAV2/1 are given. No transduction is observed with AAV2. (b) Quantification by ELISA of hPALP activity in lung homogenates 4 weeks after vector delivery. The specific serotypes and dilution factors used are shown at the bottom. Values shown are mean \pm SD. Equivalent results were obtained with the chemiluminescent assay (not shown).

Bronchoalveolar lavage was performed using two aliquots of $500 \mu \mathrm{l}$ of sterile PBS instilled through a 21gauge catheter into the trachea after exposure via a midline neck incision. The fluid was immediately aspirated, and the aliquots were pooled for analysis of FIX secretion.

Determination of serum-neutralizing antibodies. In order to identify neutralizing antibodies, serum samples $(10 \mu \mathrm{l})$ were plated in 96-well plates. Eight serial dilutions of each sample were made in serum-free DMEM. AAV2/5$\mathrm{CMV}$-enhanced green fluorescent protein (CMV-EGFP) $\left(1 \times 10^{10} \mathrm{GCs}\right)$ was added to each well for neutralizing antibody assays. The plates were incubated for 1 hour at $37^{\circ} \mathrm{C}$, and then the samples were transferred to 96-well flat-bottom tissue culture plates, which had been seeded overnight with $2.5 \times 10^{4} 84-31$ cells ( 293 cells that express the $\mathrm{E} 3$ and $\mathrm{E} 4$ genes). Cells were incubated with virus for 48 hours. At that point, plates were inspected visually and EGFP-positive cells were counted. The titer of neutralizing antibody is defined as the highest dilution at which less than $50 \%$ of the cells express EGFP.

\section{Results}

Comparison of lung transduction efficiency of $A A V$ serotypes. To compare the ability of recombinant vectors based on different AAV serotypes to transduce the lung, we packaged hybrid recombinant viruses containing an AAV2 genome encoding for hPALP in virions formed by capsids of AAV1, 2, or 5 (AAV2/1-, AAV2/2-, or AAV2/5-CB-hPALP). Intranasal administration of $50 \mu \mathrm{l}$ of vector solution

\section{Figure 2}

Serum Epo (a) and hematocrit (b) levels following intranasal administration of vectors based on various AAV serotypes in mice. We intranasally administered $3 \times 10^{11}$ GCs per mouse of AAV2/1-, AAV2/2-, or AAV2/5-CC10-rhEpo 4 weeks before the measurements. Control animals received the same set of vectors containing lacZ. Values shown are mean \pm SD. containing either $3 \times 10^{11}(1: 1)$ or $6 \times 10^{10}(1: 5)$ GCs of each vector serotype to C57BL/ 6 mice ( $n=5$ per group) resulted in transduction of alveolar and, to a lesser extent, airway surface epithelial cells, consistent with previous reports (13). Expression was higher with AAV2/5 than AAV2/1, and AAV2/2-mediated gene transfer was negligible (Figure 1a). Quantification of alkaline phosphatase in lung homogenates from the same animals confirmed the histochemical results (Figure 1b). Therefore, $\mathrm{AAV}$ vectors containing the capsid from serotype 5 seem most promising in the context of gene therapies in which targeting the intrapulmonary epithelia is desired. Secretion of Epo from lung transduced with AAV serotypes. We explored the ability of AAV vectors administered

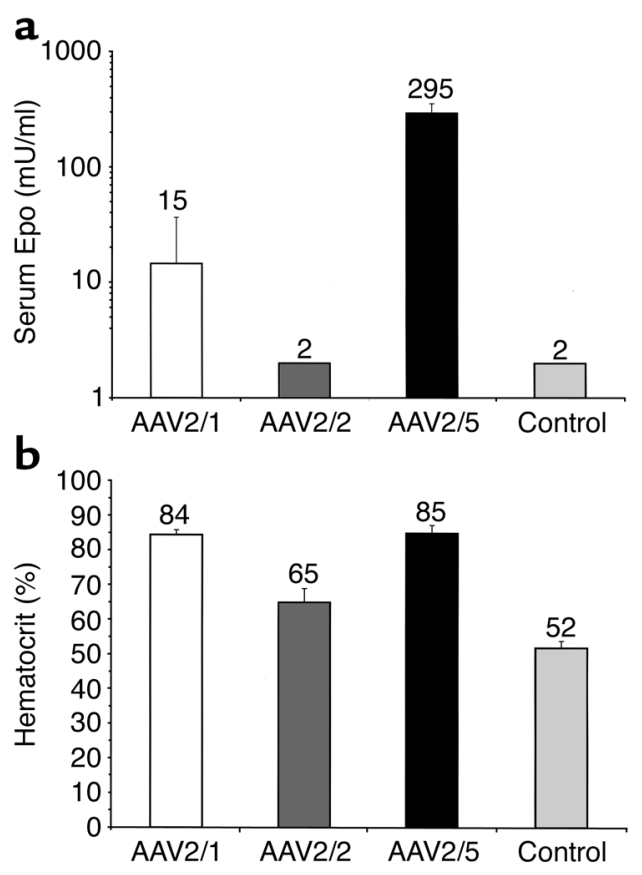



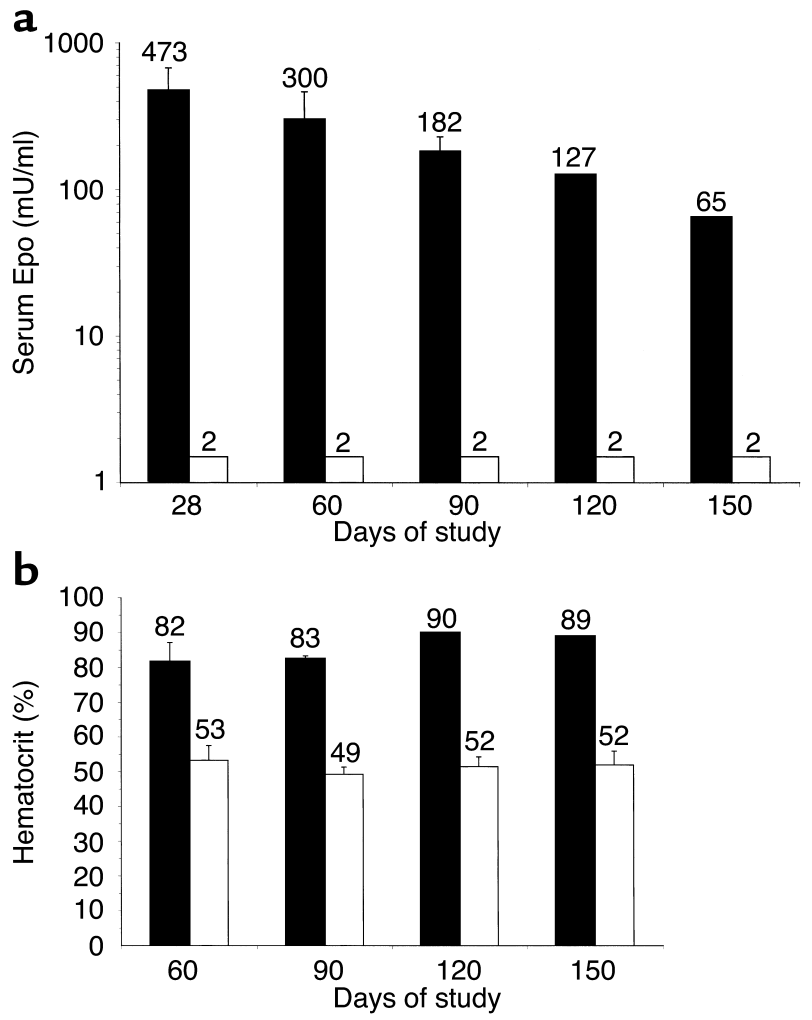

intranasally to mediate secretion of therapeutic proteins in the systemic circulation. To ensure that expression from inhaled vectors occurred as a result of transduction of lung epithelial cells alone, we used an expression cassette containing the first $320 \mathrm{bp}$ of the rat CC10 gene promoter (18), which restricts gene expression to lung epithelial cells. Vectors expressing rhEpo were created based on packaging with AAV1, AAV2, and AAV5 capsids and administered intranasally to nude mice ( $n=5$ per group, $3 \times 10^{11} \mathrm{GCs}$ of each virus per mouse). Circulating Epo levels were highest following gene transfer with AAV2/5 vectors (Figure 2a). As a result of expression of functional Epo, hematocrit levels increased most significantly in the groups given AAV2/5 and AAV2/1 and increased slightly in the group given AAV2/2 (Figure $2 \mathrm{~b}$ ). The slight hematocrit increment from AAV2-dosed animals suggests that hormone release below the detection limit of the ELISA was occurring from the targeted lung. Control animals that received AAV2/1, 2/2, and $2 / 5$ expressing the reporter gene lac $Z$ from the CC10 promoter were sacrificed at day 60 , and lungs were sectioned and stained for $\beta$-galactosidase activity. The pattern and levels of

\section{Figure 4}

FIX secretion from $\mathrm{FIX}^{-1-}$ murine lung transduced with AAV2/5. (a) Circulating FIX levels over time following viral delivery. White bars correspond to animals that received AAV2/5-CC10-lacZ, black bars to animals that were given AAV2/5-CC10-cFIX. (b) aPTT levels following intranasal delivery of AAV2/5-CC10-lacZ (white bars) or AAV2/5-CC10-FIX (black bars). Values shown are mean \pm SD.

\section{Figure 3}

Serum Epo (a) and hematocrit (b) levels over time following a single intranasal administration of $3 \times 10^{11} \mathrm{GCs}$ per mouse of AAV2/ 5-CC10-rhEpo (black bars) or AAV2/5-CC10-lacZ (white bars). Values shown are mean \pm SD (at 120 and 150 days, all but two animals had died due to effects of severe polycythemia).

gene expression were similar to those represented in Figure 1 for the alkaline phosphatase reporter gene and to those in animals receiving intranasal AAV2/5-CMVlacZ (data not shown). The levels of circulating Epo and hematocrits remained high, although there was a slow decline of Epo levels over 150 days following intranasal administration of AAV2/5 (Figure 3).

Secretion of FIX from lung transduced with $A A V 2 / 5$. We administered AAV2/5-CC10-cFIX (encoding cFIX) or AAV2/5-CC10-lacZ (control) intranasally to $F I X^{-/-}$ C57BL $/ 6$ mice ( $n=4$ per group) and followed the levels of circulating FIX. Serum FIX increased to $107 \mathrm{ng} / \mathrm{ml}$ over 45 days (Figure 4a). These levels remained elevated $(100 \pm 63 \mathrm{ng} / \mathrm{ml})$ at 288 days after vector administration. FIX was also detectable in the bronchoalveolar lavage of four animals sacrificed 288 days after noninvasive administration of vector $(262 \pm 121 \mathrm{ng} / \mathrm{ml})$. No FIX was detected in animals given AAV2/5-CC10-lacZ (data not shown). To test whether the recombinant protein secreted from the lung was functional, we measured the aPTT; we found it to be significantly reduced in the animals that received the FIX vector compared with the control vector-treated animals (Figure 4b). The biological response of vector-encoded FIX was clearly detectable, although the levels obtained may not be curative in humans.

Readministration of $A A V 2 / 5$ to murine lung. Since gene expression from transduced lungs seems to decrease significantly 5 months after vector delivery, we tested whether AAV2/5 readministration to the lung is

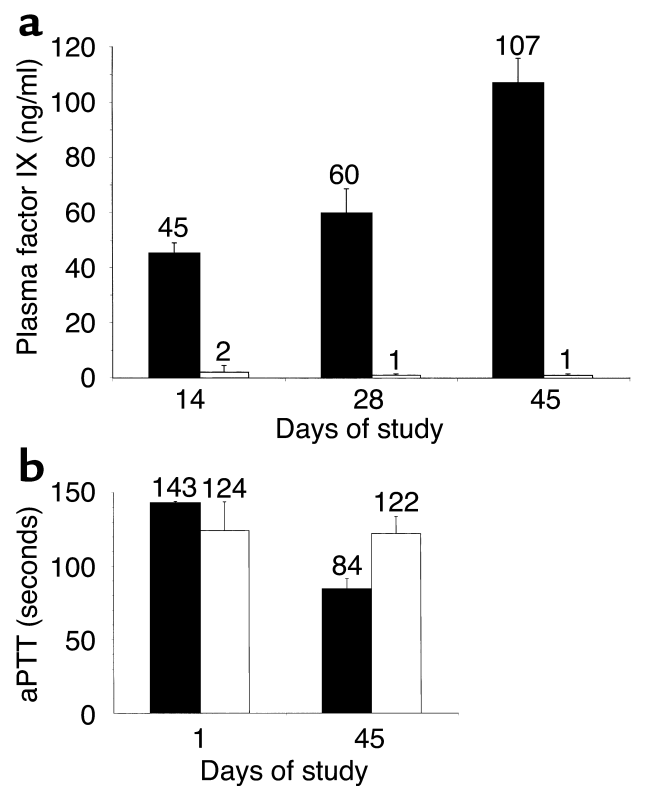


feasible. FIX $X^{-/-}$mice (7 months old) either untreated or treated 5 months before with one intranasal administration of AAV2/5-CC10-cFIX (the same animals used for the experiment shown in Figure 4) received $3 \times 10^{11}$ GCs per animal of AAV2/5-CC10lacZ intranasally. $\beta$-Galactosidase activity was measured in transduced lungs 4 weeks later and did not differ significantly between the naive and the previously treated animals (naive mice: $470 \pm 194 \mathrm{pg} / \mathrm{ml}$; previously treated mice: $416 \pm 209 \mathrm{pg} / \mathrm{ml} ; n=3$ animals per group). These results suggest that in the lung, unlike in other tissues, readministration of AAV2 $/ 5$ is feasible, potentially allowing for repeated, successful treatment of chronic diseases. The immunologic mechanisms by which this occurs were explored by analyzing immunocompetent animals $(n=5, \mathrm{C} 57 \mathrm{BL} / 6)$ administered a hybrid vector (AAV2/5-CC10-lacZ) intranasally 288 days earlier. All animals showed serum-neutralizing antibodies to AAV5 capsid protein (1:160-1:320).

\section{Discussion}

The lung, like the gastrointestinal tract, offers a large surface area with good vascularization and immense capacity for solute exchange. The lung has been used for systemic delivery of protein and peptides (e.g., insulin) via noninvasive pulmonary administration. Gene transfer is one technique by which pulmonary delivery of therapeutic proteins might be accomplished.

Using AAV hybrid vectors with the same genome, we were able to compare side by side the effect of different capsid proteins on lung transduction. To ensure that gene expression following noninvasive intranasal administration of our vectors was the result of epithelial transduction, we used a lung-specific promoter. We chose a 320-bp fragment of the CC10 promoter. To examine the specificity of this promoter fragment, we compared expression of vectors using this $320-b p$ CC10 promoter fragment with expression of vectors using a CMV promoter in 293 cells and found the former to be almost undetectable, as expected (data not shown). In vivo, we showed that the 320-bp CC10 promoter fragment resulted in expression in both alveolar and airway epithelial cells, similar to that observed in transgenic animals (18). In addition, this promoter fragment resulted in very similar lung expression to that achieved with the CMV promoter (data not shown), suggesting that the CC10 promoter fragment is as potent a promoter in the lung as is the less cell-specific CMV promoter.

We show that, following intranasal administration of our vectors, AAV5 is superior to AAV1 gene transfer and AAV2 gene transfer results in negligible transduction in the murine lung. This comparison expands on previous results demonstrating that AAV5 and AAV1 are superior to AAV2 in transducing airway epithelial cells $(13,16)$. Additionally, the superiority of AAV2/5 in the lung is confirmed using two different transgenes (alkaline phosphatase and Epo) and by multiple techniques. In contrast, comparative experiments in muscle have shown that AAV2/ 1 is most efficient (28). These results highlight that comparison of alternative AAV serotypes may allow for efficient targeting of specific organs or tissues.

Following a single intranasal administration of AAV2/5-CC10-rhEpo, sustained expression of Epo was observed for the 150 days of the study. Interestingly, Epo levels start to decrease 90 days after infection, presumably reflecting normal turnover of the epithelial cells, but possibly also reflecting gene inactivation. Hematocrit levels were consistently increased for the length of the experiment, due to the high sensitivity of the bone marrow hematopoietic compartment to even low levels of circulating Epo.

These results suggest that the lung can be used as a depot for the systemic release of therapeutic proteins following noninvasive administration of vectors. We tested this approach in a disease model that represents a traditional target of gene therapeutics, hemophilia B (29). Promising results in murine $(20,30)$ and canine (31-35) models of the disease have been obtained following muscle and liver transduction with AAV and have prompted the first clinical trial using an AAV2based vector expressing FIX from the muscle of hemophiliac patients $(36,37)$.

Following noninvasive administration of AAV2/5CC10-FIX to murine lung, secretion of functional FIX in the blood was observed. Although we did not achieve levels in mice that would correlate with normal serum FIX levels in humans, it is presumed that only a fraction of normal protein levels is required for physiologic correction of hemophilia (38). In fact, current prophylactic approaches for patients with hemophilia B aim to achieve $1 \%$ normal activity (39), which is extrapolated to correlate with approximately $16-60 \mathrm{ng} / \mathrm{ml}$ of FIX (36). Data extrapolated from animal studies would suggest that our achieved plasma FIX levels (mean of $107 \mathrm{ng} / \mathrm{ml}$ at day 45 ) correlate with $1.2-1.8 \%$ of normal FIX activity in humans (36), which is in the range of the goal of current transfusion regimens. The FIX secreted from AAV-transduced lung is clearly functional, as demonstrated by a significantly reduced (although not completely normalized [ref. 25]) aPTT. The presence of FIX in the bronchoalveolar lavage suggests that secretion of recombinant protein occurs apically as well as basolaterally in these polarized cells.

Our data demonstrate that AAV2/5 can be successfully readministered to the mouse lung, obtaining levels of gene expression comparable to those in naive mice. The presence of serum-neutralizing antibodies to AAV5 capsid proteins almost 10 months after vector administration suggests that lung transduction may occur despite the presence of serum antibodies. We did not examine the kinetics of the humoral immune response, although presumably titers in the animals administered AAV2/5-CC10-cFIX were at least as high at 5 months (the time point at which readministration was successful). Previous data suggest that successful 
readministration of $A A V$-based vectors requires the use of alternate AAV serotypes $(12,22)$ or transient immunosuppression (40), which would be impractical in the clinical setting. Further study of the humoral and cellular immune responses to these hybrid vectors is warranted. The ability to readminister the same serotype of AAV five months after the initial administration is encouraging, since readministration would be critical for treatment of chronic diseases.

In conclusion, we show that $A A V$ vectors containing the capsid from serotype 5 transduce lung epithelial cells more efficiently than do AAV2/1 and AAV2/2, and that AAV $2 / 5$ can be noninvasively readministered. Furthermore, AAV2/5-mediated pulmonary transduction results in systemic release of functional Epo and FIX. This suggests that the lung may serve as an important depot for the release of secreted protein following its noninvasive transduction.

\section{Acknowledgments}

D. Weiner is funded by the Harry Shwachman Clinical Investigator Award from the Cystic Fibrosis Foundation. J.M. Wilson is funded by the Cystic Fibrosis Foundation, Juvenile Diabetes Research Foundation International, NIH (P30 DK47757-07, P01 HL59407-02), and GlaxoSmithKline. J.M. Wilson holds equity in Targeted Genetics Corp. Assistance from the Vector and Cell Morphology Cores of the Institute for Human Gene Therapy is gratefully acknowledged.

1. Agu, R.U., Ugwoke, M.I., Armand, M., Kinget, R., and Verbeke, N. 2001. The lung as a route for systemic delivery of therapeutic proteins and peptides. Respir. Res. 2:198-209.

2. Rosenfeld, M.A., et al. 1991. Adenovirus-mediated transfer of a recombinant alpha 1-antitrypsin gene to the lung epithelium in vivo. Science. 252:431-434.

3. Flotte, T.R., et al. 1993. Stable in vivo expression of the cystic fibrosis transmembrane conductance regulator with an adeno-associated virus vector. Proc. Natl. Acad. Sci. USA. 90:10613-10617.

4. Kobinger, G.P., Weiner, D.J., Yu, Q.C., and Wilson, J.M. 2001. Filoviruspseudotyped lentiviral vector can efficiently and stably transduce airway epithelia in vivo. Nat. Biotechnol. 19:225-230.

5. Monahan, P.E., and Samulski, R.J. 2000. Adeno-associated virus vectors for gene therapy: more pros than cons? Mol. Med. Today. 6:433-440.

6. Xiao, W., et al. 1999. Gene therapy vectors based on adeno-associated virus type 1. J. Virol. 73:3994-4003.

7. Senapathy, P., and Carter, B.J. 1984. Molecular cloning of adeno-associated virus variant genomes and generation of infectious virus by recombination in mammalian cells. J. Biol. Chem. 259:4661-4666.

8. Samulski, R.J., Berns, K.I., Tan, M., and Muzyczka, N. 1982. Cloning of adeno-associated virus into pBR322: rescue of intact virus from the recombinant plasmid in human cells. Proc. Natl. Acad. Sci. USA. 79:2077-2081.

9. Chiorini, J.A., Kim, F., Yang, L., and Kotin, R.M. 1999. Cloning and characterization of adeno-associated virus type 5. J. Virol. 73:1309-1319.

10. Chiorini, J.A., Yang, L., Liu, Y., Safer, B., and Kotin, R.M. 1997. Cloning of adeno-associated virus type 4 (AAV4) and generation of recombinant AAV4 particles. J. Virol. 71:6823-6833.

11. Rutledge, E.A., Halbert, C.L., and Russell, D.W. 1998. Infectious clones and vectors derived from adeno-associated virus (AAV) serotypes other than AAV type 2. J. Virol. 72:309-319.

12. Halbert, C.L., Rutledge, E.A., Allen, J.M., Russell, D.W., and Miller, A.D. 2000. Repeat transduction in the mouse lung by using adeno-associated virus vectors with different serotypes. J. Virol. 74:1524-1532.

13. Halbert, C.L., Allen, J.M., and Miller, A.D. 2001. Adeno-associated virus type 6 (AAV6) vectors mediate efficient transduction of airway epithelial cells in mouse lungs compared to that of AAV2 vectors. J. Virol. 75:6615-6624.
14. Bals, R., et al. 1999. Transduction of well-differentiated airway epithelium by recombinant adeno-associated virus is limited by vector entry. J. Virol. 73:6085-6088.

15. Duan, D., Yue, Y., Yan, Z., Yang, J., and Engelhardt, J.F. 2000. Endosomal processing limits gene transfer to polarized airway epithelia by adenoassociated virus. J. Clin. Invest. 105:1573-1587.

16. Zabner, J., et al. 2000. Adeno-associated virus type 5 (AAV5) but not AAV2 binds to the apical surfaces of airway epithelia and facilitates gene transfer. J. Virol. 74:3852-3858.

17. Walters, R.W., et al. 2001. Binding of adeno-associated virus type 5 to 2,3linked sialic acid is required for gene transfer. J. Biol. Chem. 276:20610-20616.

18. Stripp, B.R., et al. 1992. cis-acting elements that confer lung epithelial cell expression of the CC10 gene. J. Biol. Chem. 267:14703-14712.

19. Auricchio, A., Hildinger, M., O’Connor, E., Gao, G.P., and Wilson, J.M. 2001. Isolation of highly infectious and pure adeno-associated virus type 2 vectors with a single-step gravity-flow column. Hum. Gene Ther. 12:71-76.

20. Wang, L., Takabe, K., Bidlingmaier, S.M., Ill, C.R., and Verma, I.M. 1999. Sustained correction of bleeding disorder in hemophilia B mice by gene therapy. Proc. Natl. Acad. Sci. USA. 96:3906-3910.

21. Fisher, K.J., et al. 1996. Transduction with recombinant adeno-associated virus for gene therapy is limited by leading-strand synthesis. J. Virol. 70:520-532.

22. Hildinger, M., et al. 2001. Hybrid vectors based on adeno-associated virus serotypes 2 and 5 for muscle-directed gene transfer. J. Virol. 75:6199-6203.

23. Gao, G., et al. 2000. Purification of recombinant adeno-associated virus vectors by column chromatography and its performance in vivo. Hum. Gene Ther. 11:2079-2091.

24. Salvetti, A., et al. 1998. Factors influencing recombinant adeno-associated virus production. Hum. Gene Ther. 9:695-706.

25. Wang, L., et al. 1997. A factor IX-deficient mouse model for hemophilia B gene therapy. Proc. Natl. Acad. Sci. USA. 94:11563-11566.

26. Fisher, K.J., et al. 1997. Recombinant adeno-associated virus for muscle directed gene therapy. Nat. Med. 3:306-312.

27. Axelrod, J.H., Read, M.S., Brinkhous, K.M., and Verma, I.M. 1990. Phenotypic correction of factor IX deficiency in skin fibroblasts of hemophilic dogs. Proc. Natl. Acad. Sci. USA. 87:5173-5177.

28. Chao, H., et al. 2000. Several log increase in therapeutic transgene delivery by distinct adeno-associated viral serotype vectors. Mol. Ther. 2:619-623.

29. High, K.A. 2001. Gene transfer as an approach to treating hemophilia. Circ. Res. 88:137-144.

30. Herzog, R.W., et al. 1997. Stable gene transfer and expression of human blood coagulation factor IX after intramuscular injection of recombinant adeno-associated virus. Proc. Natl. Acad. Sci. USA. 94:5804-5809.

31. Herzog, R.W., Mount, J.D., Arruda, V.R., High, K.A., and Lothrop, C.D., Jr. 2001. Muscle-directed gene transfer and transient immune suppression result in sustained partial correction of canine hemophilia B caused by a null mutation. Mol. Ther. 4:192-200.

32. Herzog, R.W., et al. 1999. Long-term correction of canine hemophilia B by gene transfer of blood coagulation factor IX mediated by adeno-associated viral vector. Nat. Med. 5:56-63.

33. Wang, L., Nichols, T.C., Read, M.S., Bellinger, D.A., and Verma, I.M. 2000. Sustained expression of therapeutic level of factor IX in hemophilia B dogs by AAV-mediated gene therapy in liver. Mol. Ther. 1:154-158.

34. Chao, H., et al. 1999. Persistent expression of canine factor IX in hemophilia B canines. Gene Ther. 6:1695-1704.

35. Monahan, P.E., et al. 1998. Direct intramuscular injection with recombinant AAV vectors results in sustained expression in a dog model of hemophilia. Gene Ther. 5:40-49.

36. Kay, M.A., et al. 2000. Evidence for gene transfer and expression of factor IX in haemophilia B patients treated with an AAV vector. Nat. Genet. 24:257-261.

37. Larson, P.J., and High, K.A. 2001. Gene therapy for hemophilia B: AAVmediated transfer of the gene for coagulation factor IX to human muscle. Adv. Exp. Med. Biol. 489:45-57.

38. Walter, J., and High, K.A. 1997. Gene therapy for the hemophilias. Adv. Vet. Med. 40:119-134.

39. Lofqvist, T., Nilsson, I.M., Berntorp, E., and Pettersson, H. 1997. Haemophilia prophylaxis in young patients: a long-term follow-up. J. Intern. Med. 241:395-400.

40. Halbert, C.L., Standaert, T.A., Wilson, C.B., and Miller, A.D. 1998. Successful readministration of adeno-associated virus vectors to the mouse lung requires transient immunosuppression during the initial exposure. J. Virol. 72:9795-9805. 